\title{
Effects of Peritumoral Nanoconjugated Cisplatin on Laryngeal Cancer Stem Cells
}

Michael W. Sim, $\mathrm{MD}^{1}$, Patrick T. Grogan, $\mathrm{PhD}^{2}$, Chitra Subramanian, $\mathrm{PhD}^{3}$, Carol R. Bradford, $\mathrm{MD}^{1}$, Thomas E. Carey, $\mathrm{PhD}^{1}, \mathrm{M}$. Laird Forrest, $\mathrm{PhD}^{4}$, Mark E. Prince, $M D^{1}$, Mark S. Cohen, $\mathrm{MD}^{3}$.

1. Department of Otolaryngology - Head and Neck Surgery, University of Michigan Health System, Ann Arbor, MI.

2. University of Kansas School of Medicine, Kansas City, KS.

3. Department of Surgery, University of Michigan Health System, Ann Arbor, MI.

4. University of Kansas School of Pharmacy, Kansas City, KS.

Running Title: Nanoconjugated Cisplatin and Cancer Stem Cells

Financial support: Funding support for this project was provided in part from the AHNS Alando J. Ballantyne Research Grant (Sim), University of Michigan Department of Surgery (Cohen), the University of Michigan Cancer Center Support Grant (Cohen), and $\mathrm{NCI}$ R01CA173292 (Forrest).

Conflict of Interest: Drs. Forrest and Cohen have financial interest in a company Hylapharm, which has licensed portions of technologies described in this study.

\section{Corresponding Author Contact Information:}

Michael W. Sim, MD 1904 Taubman Center 1500 E. Medical Center Dr. Ann Arbor, Ml 48109 michaelsim@gmail.com (734) 748-5649

Presentation: This study was presented as an oral presentation at the IFHNOS 5th World Congress/AHNS 2014 meeting held July $27-30$ in New York City, NY.

This is the author manuscript accepted for publication and has undergone full peer review but has not been through the copyediting, typesetting, pagination and proofreading process, which may lead to differences between this version and the Version record. Please cite this article as doi:10.1002/ lary.25808. 


\section{ABSTRACT}

Objective: To evaluate the efficacy of peritumoral hyaluronic acid-cisplatin therapy in a murine model of laryngeal squamous cell carcinoma and to evaluate its effect on cancer stem cells.

Study Design: An orthotopic murine study utilizing UMSCC-12 laryngeal cancer cells was conducted in randomized controlled fashion with three treatment arms: saline, systemic cisplatin, and peritumoral HA-cisplatin.

Methods: UMSCC-12 laryngeal cancer cells were inoculated into the buccal mucosa of athymic nude mice followed by weekly treatment with saline, systemic cisplatin, or peritumoral HA-cisplatin for 3 weeks. Tumor response and animal weight was monitored and change in CD44 proportion was analyzed ex vivo.

Results: HA-cisplatin demonstrated superior antitumor efficacy and greater reduction in CD44 positivity on ex vivo analysis.

Conclusion: Peritumoral nanoconjugated HA-cisplatin provides superior antitumor efficacy compared to standard cisplatin therapy in an in vivo laryngeal cancer model. There was also selective targeting of CD44+ cancer cells with HA-cisplatin. This therapeutic strategy could represent the first selective laryngeal CSC-targeted therapy. Further preclinical investigation is warranted to evaluate its role for locally advanced head and neck cancer treatment.

Key Words: Larynx cancer, cancer stem cells, targeted therapy, CD44, hyaluronan nanoconjugate.

\section{Level of Evidence: NA}




\section{INTRODUCTION}

More than 55,000 Americans are expected to be diagnosed with head and neck cancers in 2014 , with a large proportion of these being squamous cell carcinomas ${ }^{1}$.

Approximately 12,000 of these oral cavity, pharyngeal, and laryngeal cancer patients will die from their disease yearly. Although the incidence of this disease in the US has been noted to be relatively stable, recent studies have shown the rates of oropharyngeal cancers associated with HPV to be rising ${ }^{1}$. These findings suggest that head and neck cancer will continue to be a prominent malignancy for treatment our in national healthcare cancer mission.

Historically, radiation therapy alone for patients with unresectable head and neck squamous cell carcinomas (HNSCC) provided unsatisfactory 5-year survival and recurrence rates. As a result, concurrent radiation and chemotherapy has now become the standard for adjuvant therapy after surgical ablation of the primary tumor and definitive treatment in select cases. Platinum-based chemotherapy, namely cisplatin, administered systemically via intravenous (IV) route remains a first line agent due to its radiosensitizing and cytotoxic effects ${ }^{2}$. Cisplatin therapy, however, continues to have significant limitations. Besides its known toxicities to the kidney, peripheral nerves and hearing, as a systemically delivered agent, it has poor penetration into the locoregional lymphatics. Only a very small fraction of the systemic dose of the drug will be taken up into the lymphatics and lymph nodes, which may in part be due to monodirectional lymphatic flow and pressure gradients in these channels ${ }^{3}$. This is a critical factor in patients with locally advanced disease where lymph node metastases are a frequent occurrence. Another major limitation is that HNSCC has also shown marked resistance 
to cisplatin in up to $40 \%$ of patients ${ }^{4}$, which has important implications as any efforts to overcome this resistance by increasing dosage has severe limitations due to the dosedependent toxicity of the drug ${ }^{5}$. Finally, cisplatin lacks specificity in targeting CSCs, considered by many to be the primary mediators of treatment failures ${ }^{6}$. Thus far, targeted therapy against $\mathrm{CSCs}^{7}$ is a relatively unexplored but critically important area of study that needs further investigation.

$\mathrm{HA}$ is an endogenous polysaccharide, with alternating D-glucuronic acid and Nacetyl D-glucosamine units, found in the extracellular matrix of connective tissues. In previously published work ${ }^{8}$, there were significantly increased lymphatic tissue concentrations of cisplatin and reduced organ toxicities with peritumoral injections of cisplatin conjugated to nanoscopic (25-100 nM) particles of HA (HA-cisplatin) compared to systemically delivered standard cisplatin therapy. In related studies investigating this drug in orthotopic murine models, HA-cisplatin was found to have significantly higher anticancer efficacy in vivo relative to conventional IV cisplatin therapy in HNSCC xenografts implanted in the buccal mucosa of the subject mice ${ }^{3,9}$.

Moreover, HA is also a highly specific ligand for the CD44 surface receptors ${ }^{10}$, and CD44 is described as a cell surface marker specific for CSCs in HNSCC. In fact, CSCs were first successfully isolated from HNSCC cell lines utilizing CD44 expression $^{11}$. The interactions between these two molecules, however, are yet to be investigated in the setting of stem-cell targeted therapy in HNSCC. Thus, the objectives of this study were to evaluate the efficacy and toxicity of peritumoral HA-cisplatin therapy compared to standard systemic cisplatin in vivo in a laryngeal cancer xenograft 
model via orthotopic tumor implantation, and to evaluate the effect of HA-cisplatin on the CD44 positive (+) HNSCC tumor population ex vivo.

\section{MATERIALS AND METHODS}

\section{Cell Culture}

UMSCC-12 human laryngeal cancer cells were grown and maintained in Dulbecco's modified Eagle's medium (DMEM; Sigma-Aldrich, St. Louis, MO) supplemented with $10 \%$ fetal bovine serum (FBS; Sigma-Aldrich, St. Louis, MO), and $1 \%$ combination of penicillin and streptomycin (Sigma-Aldrich, St. Louis, MO) in $37^{\circ} \mathrm{C}$ humidified atmosphere of $5 \% \mathrm{CO} 2$ in air. To utilize the cells for the various experiments, they were trypsinized using $0.25 \%$ trypsin (Sigma-Aldrich, St. Louis, MO), collected, and counted with a hemocytometer to determine the number of viable cells.

\section{In Vitro CD44 Analysis}

In vitro analysis was performed prior to conducting the in vivo study to ensure that the UMSCC-12 cell line had a relatively high CD44+ proportion of cells. To determine the CD44+ proportion, the cells were first trypsinized, collected, rinsed in 1x PBS, and placed in single-cell suspension. They were then treated with fluorochrome-conjugated CD44 antibody (BD Biosciences, San Diego, CA) for 30 minutes on ice. Once completed, the cells were rinsed again in 1x PBS and resuspended before being analyzed via flow cytometry (Beckman Coulter CyAn ADP analyzer, Brea, CA).

\section{Cell Viability Assay}


To examine the in vitro effect of HA-cisplatin and standard cisplatin on the viability and proliferation of UMSCC-12 cells, a standard MTS assay was performed. Cells were seeded in 96 -well plates at a density of 1000 cells per well. After a 24 hour incubation period, HA-cisplatin and cisplatin were added to each well in varying concentrations and then incubated for 72 hours. Cell viability was then quantified by the colorimetric CellTiter96 Aqueous MTS assay (Promega, Fitchburg, WI) at $490 \mathrm{~nm}$ on a BioTek Synergy 2 plate reader (BioTek, Winooski, VT) according to the manufacturer's instructions.

\section{In Vivo Tumor Model}

UMSCC 12 cells were allowed to proliferate in cell culture as previously described and then inoculated in the buccal mucosa of 33 athymic nude female mice 4 to 6 weeks in age (Harlan Laboratories, Inc., Indianapolis, IN). The cells were prepared in 1x PBS solution at a concentration of $1 \times 10^{6}$ cells per $100 \mu \mathrm{L}$. Tumor inoculation was performed under isoflurane anesthesia using a 25-gauge needle. Tumor size and body weight were then measured 3 times weekly with a digital caliper. Tumor volume measured in cubic millimeters was calculated using the following equation:

$$
\text { Tumor Volume }=(\pi / 6) \times(\text { Width })^{2} \times \text { Length }
$$

Body weight was measured in grams using a standard digital scale. Once tumor volumes reached $50 \mathrm{~mm}^{3}$, the mice were randomized into three treatment arms with 11 mice in each arm: control, systemic cisplatin, and peritumoral HA-cisplatin. Subjects were treated weekly at 50\% maximum tolerated dose (MTD) for the two treatment arms and with PBS for the control arm for 3 weeks. Pharmaceutical-grade cisplatin was 
utilized for both the systemic group and in the HA-cisplatin group, with the latter nanoconjugated formulation synthesized as described in previously published work $^{12}$. Treatments in the control and standard cisplatin arms were administered intraperitoneally and treatments in the HA-cisplatin group were administered subcutaneously around the tumor site. The MTD level used was $10 \mathrm{mg} / \mathrm{kg}$ per week as previously reported in studies where intraperitoneal cisplatin was dosed in mice ${ }^{13,14}$.

Once the treatment period concluded, 3 mice from each arm, designated as the early post-treatment group, were then euthanized, the tumors were harvested, and ex vivo CD44 analysis of the samples were performed. The remaining subjects were then monitored for 9 weeks post-treatment for tumor size and body weight. At end-of-study, 4 mice from each arm were analyzed for CD44+ cell proportion in the tumors ex vivo.

\section{Ex Vivo CD44 Analysis}

Harvested tumors were prepared for CD44 analysis via either Western blotting or flow cytometry. For Western blot analysis, proteins were collected, quantified, separated by sodium dodecyl sulfate-polyacrylamide gel electrophoresis (SDS-PAGE), and electrotransfered onto a Hybond nitrocellulose membrane as described in a prior study $^{15}$. Actin levels were assessed to ensure relatively equal protein loading and transfer among the tumor samples. Primary mouse antibodies included anti-CD44 antibody (\#5640; 1:1,000; Cell Signaling Technology, Beverly, MA) and anti-actin antibody (MAB1501; 1:50,000; EMD Millipore, Billerica, MA). Secondary anti-mouse antibody staining was then applied at 1:5,000 for anti-CD44 antibody and 1:10,000 for anti-actin antibody. Both the early post-treatment and end-of-study tumor samples were analyzed via Western blot. 
For flow cytometry analysis of CD44 proportion, the tumor samples were processed using a detailed tumor digestion protocol available via the University of Michigan Comprehensive Cancer Center Wicha Lab manual available online ${ }^{16}$. The main digestive agent utilized was 10x collagenase/hyaluronidase (STEMCELL Technologies Inc., Vancouver, BC, Canada) diluted 1:10 in Medium 199 (Sigma-Aldrich, St. Louis, MO). The resultant tumor cells were then converted to single-cell suspensions and treated with fluorochrome-conjugated CD44 antibody (BD Biosciences, San Diego, CA) and then analyzed via flow cytometry (Beckman Coulter CyAn ADP analyzer, Brea, CA) for CD44+ cell proportion. Of note, only the end-of-study tumor samples were large enough to be processed for flow cytometry and Western blotting.

\section{Statistical Analysis}

GraphPad Prism 6 (version 6.02; GraphPad Inc., San Diego, CA) was used to generate best-fit non-linear dose response curves for IC50 determination for the MTS assay data. Comparisons of differences between two means were determined by Student's unpaired t-test via the statistical functions of Microsoft Excel 2010 software (Microsoft Corporation, Redmond, WA). Data are presented as mean values with error bars denoting standard deviation. The level of significance was set at $p<0.05$.

\section{RESULTS}

\section{In Vitro Analysis of UMSCC-12 Cell Line}

Flow cytometry analysis showed the CD44+ proportion of cells in UMSCC-12 to be on average $61 \pm 6 \%$ in this cell population when grown in vitro (Figure 1). The relatively high proportion of CD44+ cells (60\%) within this cell line validated its use in the in vivo 
model to ensure that a knockdown effect on CD44 expression could be clearly detected with drug treatment if such an effect occurred. To evaluate the cytotoxic effect of HAcisplatin on UMSCC-12 cells, the cells were plated and treated with increasing concentrations of HA-cisplatin as well as standard cisplatin for 72 hours with resultant cell viability determined by MTS assay (Figure 2). A dose dependent decrease in cell viability was seen with both drugs demonstrating that conjugation to HA does not inhibit the cytotoxic effect of cisplatin (as previously described) ${ }^{13}$. GraphPad was used to determine the $I C_{50}$ values of the two drugs. $I C_{50}$ values were comparable between the two treatment drugs at $3.01 \pm 0.59 \mu \mathrm{g} / \mathrm{mL}$ for cisplatin and $5.59 \pm 0.76 \mu \mathrm{g} / \mathrm{mL}$ for HAcisplatin $(p=0.3)$.

\section{In Vivo Analysis of Efficacy and Toxicity}

To assess the overall efficacy of HA-cisplatin in vivo, an orthotopic xenograft murine model was utilized. Three separate treatment arms were set in randomized, controlled manner and these included control, standard cisplatin, and HA-cisplatin, with 11 mice in each group from the start. After 3 weeks of treatment, an early post-treatment group of 3 mice from each arm were euthanized and their tumors were analyzed for CD44+ proportion. The remaining mice were then monitored three times a week for tumor volume and body weight for 9 weeks at which point the study was concluded. HAcisplatin showed superior antitumor efficacy compared to cisplatin as shown by tumor volumes at 4 weeks post-treatment $\left(39.2 \mathrm{~mm}^{3}\right.$ vs. $\left.57.6 \mathrm{~mm}^{3}, \mathrm{p}=0.02\right)$ but at end-ofstudy the difference was not significant $\left(77.5 \mathrm{~mm}\right.$ vs. $\left.105.6 \mathrm{~mm}^{3}, p=0.05\right)$ (Figure 3 ). Tumor volumes in the control group demonstrated an exponential growth curve approximately 4 weeks after completion of treatment. The tumor volumes in both 
treatment arms, in contrast, were initially more stable followed by a shallow linear growth rate. One study subject in the HA-cisplatin arm experienced a complete response (CR) and one had a sustained partial response (PR) (Figure 4). None of the standard cisplatin mice had CR or PR. In evaluating toxicity, animal weights were found to be similar between the cisplatin and HA-cisplatin arms at $24.7 \pm 0.69$ grams and $24.5 \pm 0.81$ grams, respectively (Figure 5 ).

\section{Ex Vivo Analysis of CD44}

In the early post-treatment group, CD44 reduction was seen in both treatment groups, but to a greater degree with HA-cisplatin as demonstrated by Western blotting (Figure 6). At end-of-study, 4 mice from each arm were then euthanized and tumors were analyzed for CD44+ proportion, both by flow cytometry and Western blotting (Figure 7 and 8). Long-term durability of this CD44 knockdown effect was seen end-of-study in the HA-cisplatin group compared to cisplatin $(8.1 \% \mathrm{CD} 44+$ vs. $23.9 \% \mathrm{CD} 44+, \mathrm{p}=0.02)$ which was similar to control $(23.9 \%$ CD $44+$ vs. $24.8 \%$ CD $44+, p=0.86)$. The flow cytometry data was confirmed by the Western blot results which clearly demonstrated reduced expression of CD44 in the HA-cisplatin group which was maintained even 8 weeks after removal of drug therapy.

\section{DISCUSSION}

Concurrent chemoradiation therapy has lead to improved locoregional disease control compared to monotherapy and is currently the standard of care for locally advanced HNSCC patients following surgery. Even with these advancements, however, survival rates for patients with HNSCC have improved little the last three decades. In efforts to 
improve outcomes, there have been several Phase III trials that implemented cisplatin in combination with one or more drugs, such as 5-fluorouracil. Some studies showed promising results, but others showed only minimal improvement in disease-free survival and locoregional control compared to patients receiving radiation therapy alone $e^{2,17,18}$.

This grim clinical picture of HNSCC supports the vital need for discovery and development of better treatments, and improved drug-delivery over traditional systemic therapy administered intravenously. A locoregionally-directed drug-delivery, such as nanoconjugated HA-cisplatin via a weekly peritumoral injection, as was utilized in this study, may be an important treatment advance in a thus far stagnant paradigm of treatment for advanced HNSCC. While localized therapy is already being used in the treatment of esophageal cancers, limb-limited melanoma with limb perfusion techniques, and hepatic cancers via (transarterial) TACE techniques ${ }^{19,20}$, this therapeutic strategy has yet to be clinically implemented in the treatment of HNSCC.

The current study demonstrates the improved in vivo efficacy of localized, peritumoral HA-cisplatin therapy compared to systemic cisplatin therapy, especially in the early post-treatment period. The difference in tumor volume between the two treatment groups, however, was not statistically significant by end-of-study. It is important to note, though, that one mouse in the HA-cisplatin arm had a complete response with no evidence of residual tumor and one other had a sustained partial response at end-of-study. No mice in the standard cisplatin arm experienced such a response. But the overall efficacy of standard cisplatin in this study utilizing a laryngeal cancer cell line is not surprising given its proven efficacy clinically in the setting of advanced laryngeal cancer treatment. In fact, in some institutions, systemic cisplatin is 
used as a chemo-selection agent to determine candidacy for organ preservation therapy as an alternative to total laryngectomy in patients with advanced laryngeal squamous cell carcinoma ${ }^{21}$.

The natural progression of HNSCCs is to preferentially spread to the locoregional lymphatic basins. Hematogenous spread is less frequent than lymphatic spread in squamous cell carcinomas ${ }^{22}$. Despite this, systemic IV therapy has remained the standard modality of treatment. Locoregional peritumoral therapy delivers drug into the lymphatic channels, directly affecting the primary tumor and its associated lymphatic basins. This could prove to be the more effective strategy against locally advanced or recurrent disease in the future. The current study did not incorporate the treatment of nodal disease to the in vivo model, mainly due to the low in vivo metastatic potential of the UMSCC-12 cell line. Previously published studies on HA-cisplatin, however, demonstrated efficacy of peritumoral HA-cisplatin therapy even at the regional nodal basins $^{3,9}$. This suggests that HA-cisplatin could prove to be an effective therapy in locoregionally advanced HNSCCs in the human translational setting with potential to even improve survival rates in this subset of patients.

Solid tumors, including HNSCCs, normally consist of a heterogeneous population of cell typès including tumor, stromal, and inflammatory cells ${ }^{6}$. Among the tumor cells, CSCs represent a small subpopulation of cells that have the unique ability to self-renew through multiple generations, regenerate cells that have both tumorigenic and nontumorigenic properties, and sustain growth of the tumor ${ }^{23-27}$. This subpopulation is responsible for most of the growth and spread of tumor, with the non-CSC population having limited to no growth potential ${ }^{11}$. Analogous to normal stem cells, CSCs can lie 
quiescent for periods of time and harbor mechanisms to protect against DNA and cellular damage ${ }^{6}$. Given these properties, it is not surprising that CSCs have been shown to have resistance against chemotherapeutic agents and external beam radiation ${ }^{28,29}$. An additional feature of CSCs is that they can be isolated from the other tumor cell types by distinctive cell surface markers, namely CD44 and ALDH in HNSCC. In fact, CSCs were first successfully isolated from HNSCC cell lines utilizing CD44 expression ${ }^{11}$. It is theorized that treatment failures are due to a failure in targeting and eliminating CSCs resulting in tumor regrowth and resistance to therapy ${ }^{30}$.

\section{Coincidentally, HA nanoconjugation, which allowed for cisplatin to be} administered via peritumoral route, also provided the potential for HA-cisplatin to preferentially target the CD44+ subpopulation, and thus, the CSCs in a tumor. The results of the current study showed significant evidence of this selective CD44-targeting by HA-cisplatin. The early post-treatment tumors showed decreased expression of CD44 in the HA-cisplatin treated group compared to cisplatin-treated mice and controls according to Western blot analysis. The end-of-study tumors showed significant reduction in CD44+ cell proportion via flow cytometry in the HA-cisplatin treated populations, and these findings correlated with the Western blot analysis. It is important to note, however, that although decrease in CD44 positivity allows one to infer effective CSC-targeting, a more accurate way to determine this would incorporate assessing for both CD44 and ALDH positivity. Overall, these results provide the preliminary basis for further exploration and development of CSC-specific therapies.

\section{CONCLUSION}


Peritumoral nanoconjugated HA-cisplatin provides superior antitumor efficacy compared to standard cisplatin therapy in an in vivo laryngeal cancer xenograft model. Moreover, it may potentially target laryngeal CSCs as evidenced by its selective effect on CD44+ cells within a heterogeneous tumor population. These results provide support for further translational investigation of this treatment modality as a potential CSCtargeting agent for future clinical application to patients with locally advanced HNSCC.

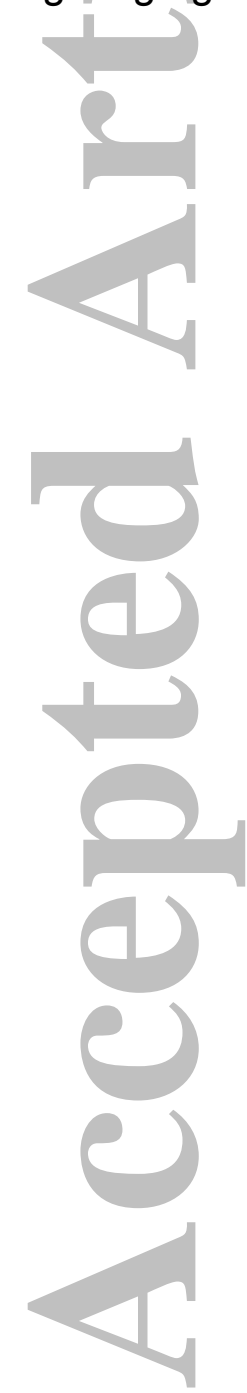

John Wiley \& Sons

This article is protected by copyright. All rights reserved. 


\section{ACKNOWLEGEMENTS}

Funding support for this project was provided in part from the AHNS Alando J. Ballantyne Research Grant (Sim), University of Michigan Department of Surgery (Cohen), the University of Michigan Cancer Center Support Grant (Cohen), and NCI R01CA173292 (Forrest).

John Wiley \& Sons

This article is protected by copyright. All rights reserved. 


\section{REFERENCES}

1. American Cancer Society (2014): Cancer Facts \& Figures 2014. Atlanta, GA, 2014.

2. Forastiere AA. Chemotherapy in the treatment of locally advanced head and neck cancer. Journal of surgical oncology 2008; 97:701-707.

3. Cai S, Xie Y, Davies NM, Cohen MS, Forrest ML. Carrier-based intralymphatic cisplatin chemotherapy for the treatment of metastatic squamous cell carcinoma of the head \& neck. Therapeutic delivery 2010; 1:237-245.

4. Kelland L. The resurgence of platinum-based cancer chemotherapy. Nature reviews Cancer 2007; 7:573-584.

5. Chu G. Cellular responses to cisplatin. The roles of DNA-binding proteins and DNA repair. The Journal of biological chemistry 1994; 269:787-790.

6. Prince ME, Ailles LE. Cancer stem cells in head and neck squamous cell cancer. Journal of clinical oncology : official journal of the American Society of Clinical Oncology 2008; 26:2871-2875.

7. Korkaya H, Wicha MS. Selective targeting of cancer stem cells: a new concept in cancer therapeutics. BioDrugs : clinical immunotherapeutics, biopharmaceuticals and gene therapy $2007 ; 21: 299-310$.

8. Cai S, Xie Y, Davies NM, Cohen MS, Forrest ML. Pharmacokinetics and disposition of a localized lymphatic polymeric hyaluronan conjugate of cisplatin in rodents. Journal of pharmaceutical sciences 2010; 99:2664-2671.

9. Cohen SM, Rockefeller N, Mukerji Ret al. Efficacy and toxicity of peritumoral delivery of nanoconjugated cisplatin in an in vivo murine model of head and neck 
squamous cell carcinoma. JAMA otolaryngology-- head \& neck surgery 2013; 139:382-387.

0. Wang SJ, Bourguignon LY. Role of hyaluronan-mediated CD44 signaling in head and neck squamous cell carcinoma progression and chemoresistance. The American journal of pathology 2011; 178:956-963.

1. Prince ME, Sivanandan R, Kaczorowski Aet al. Identification of a subpopulation of cells with cancer stem cell properties in head and neck squamous cell carcinoma. Proceedings of the National Academy of Sciences of the United States of America 2007; 104:973-978.

2. Cai S, Xie Y, Bagby TR, Cohen MS, Forrest ML. Intralymphatic chemotherapy using a hyaluronan-cisplatin conjugate. The Journal of surgical research 2008; $147: 247-252$.

3. Feleszko W, Zagozdzon R, Golab J, Jakobisiak M. Potentiated antitumour effects of cisplatin and lovastatin against MmB16 melanoma in mice. Eur J Cancer 1998; $34: 406-411$.

4. Graeser R, Esser N, Unger Het al. INNO-206, the (6-maleimidocaproyl hydrazone derivative of doxorubicin), shows superior antitumor efficacy compared to doxorubicin in different tumor xenograft models and in an orthotopic pancreas carcinoma model. Invest New Drug 2010; 28:14-19.

5. Samadi AK, Zhang X, Mukerji R, Donnelly AC, Blagg BS, Cohen MS. A novel Cterminal HSP90 inhibitor KU135 induces apoptosis and cell cycle arrest in melanoma cells. Cancer letters 2011; 312:158-167. 
6. Wicha Lab Manual, University of Michigan Comprehensive Cancer Center. Available at: http://www.med.umich.edu/wicha-lab/SOP/SOP\%202.2\%20Human\%20Tumor\%20Digestion.pdf.

7. Chandana SR, Conley BA. Neoadjuvant chemotherapy for locally advanced squamous cancers of the head and neck: current status and future prospects.

Current opinion in oncology 2009; 21:218-223.

8. Jacobs C, Lyman G, Velez-Garcia Eet al. A phase III randomized study comparing cisplatin and fluorouracil as single agents and in combination for advanced squamous cell carcinoma of the head and neck. Journal of clinical oncology : official journal of the American Society of Clinical Oncology 1992; 10:257-263.

9. Noda $\mathrm{T}$, Ohigashi $\mathrm{H}$, Ishikawa Oet al. Liver perfusion chemotherapy for selected patients at a high-risk of liver metastasis after resection of duodenal and ampullary cancers. Annals of surgery $2007 ; 246: 799-805$.

0. Sanki A, Kam PC, Thompson JF. Long-term results of hyperthermic, isolated limb perfusion for melanoma: a reflection of tumor biology. Annals of surgery 2007; 245:591-596.

1. Worden FP, Moyer J, Lee JSet al. Chemoselection as a strategy for organ preservation in patients with T4 laryngeal squamous cell carcinoma with cartilage invasion. The Laryngoscope 2009; 119:1510-1517.

2. Wong SY, Hynes RO. Lymphatic or hematogenous dissemination: how does a metastatic tumor cell decide? Cell cycle 2006; 5:812-817. 
3. Al-Hajj M, Clarke MF. Self-renewal and solid tumor stem cells. Oncogene 2004; 23:7274-7282.

4. Cho RW, Clarke MF. Recent advances in cancer stem cells. Current opinion in genetics \& development 2008; 18:48-53.

5. Lobo NA, Shimono Y, Qian D, Clarke MF. The biology of cancer stem cells.

Annual review of cell and developmental biology 2007; 23:675-699.

6. Reya T, Morrison SJ, Clarke MF, Weissman IL. Stem cells, cancer, and cancer stem cells. Nature $2001 ; 414: 105-111$.

7. Wicha MS, Liu S, Dontu G. Cancer stem cells: an old idea--a paradigm shift. Cancer research 2006; 66:1883-1890; discussion 1895-1886.

8. Al-Assar O, Muschel RJ, Mantoni TS, McKenna WG, Brunner TB. Radiation response of cancer stem-like cells from established human cell lines after sorting for surface markers. International journal of radiation oncology, biology, physics 2009; 75:1216-1225.

9. Bao S, Wu Q, McLendon REet al. Glioma stem cells promote radioresistance by preferential activation of the DNA damage response. Nature 2006; 444:756-760.

0. Aschenko I, Seeliger H, Schaffer M, Jauch KW, Bruns CJ. Cancer stem cells: how can we target them? Current medicinal chemistry 2008; 15:3171-3184. 


\section{FIGURE LEGENDS}

Figure 1. One sample of CD44+ cell proportion in UMSCC-12 cell culture population in vitro as determined by flow cytometry.

Figure 2. Best-fit non-linear dose response curves showing dose-dependent decrease in cell viability of UMSCC-12 cells with drug treatment.

Figure 3. Tumor response curves for the three treatment arms.

Figure 4. Examples of subject mice. A, a cisplatin mouse early after completion of treatment. B, a HA-cisplatin mouse with partial response. C, a control mouse showing uninhibited tumor growth.

Figure 5. Weights of animal subjects monitored throughout the study.

Figure 6. Western blot from early post-treatment group demonstrating reduction of CD44 expression in the two treatment arms, but to greater degree in HA-cisplatin.

Figure 7. A, Flow cytometry data from end-of-study group demonstrating significant reduction of CD44 expression in the HA-cisplatin. B, Examples of flow cytometry diagrams from each group. 
Figure 8. Western blot from end-of-study group demonstrating reduction of CD44 expression in HA-cisplatin group.
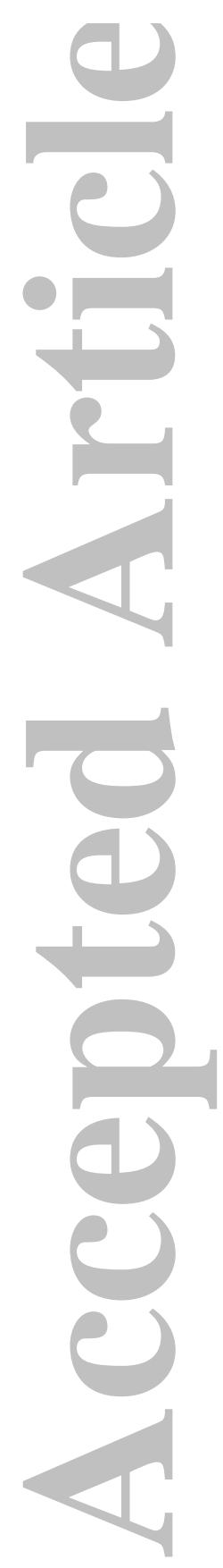

John Wiley \& Sons

This article is protected by copyright. All rights reserved. 


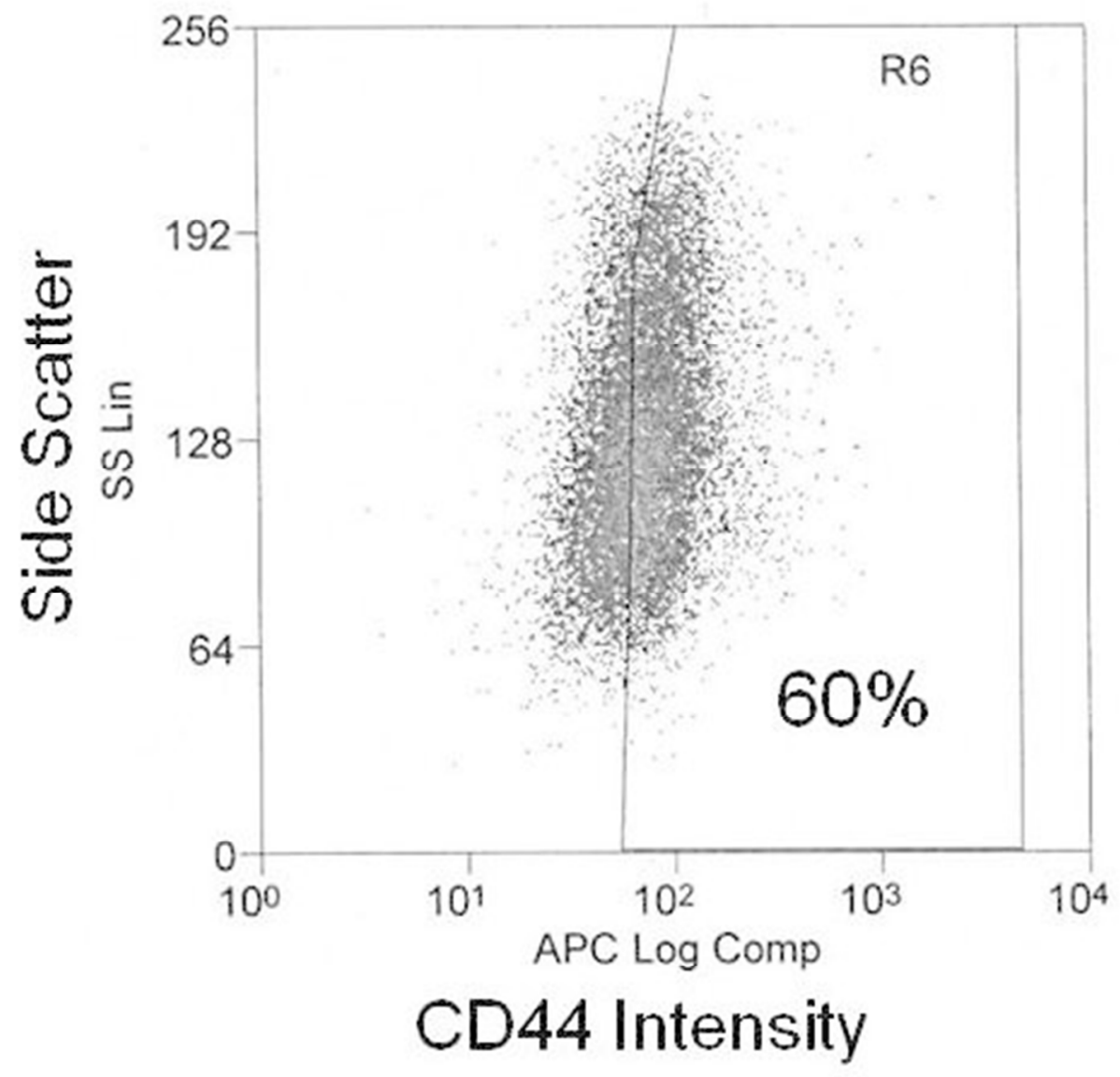

Figure 1. One sample of $\mathrm{CD} 44+$ cell proportion in UMSCC-12 cell culture population in vitro as determined by flow cytometry.

$85 \times 87 \mathrm{~mm}(150 \times 150 \mathrm{DPI})$

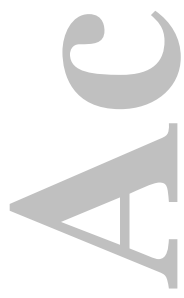

John Wiley \& Sons

This article is protected by copyright. All rights reserved. 


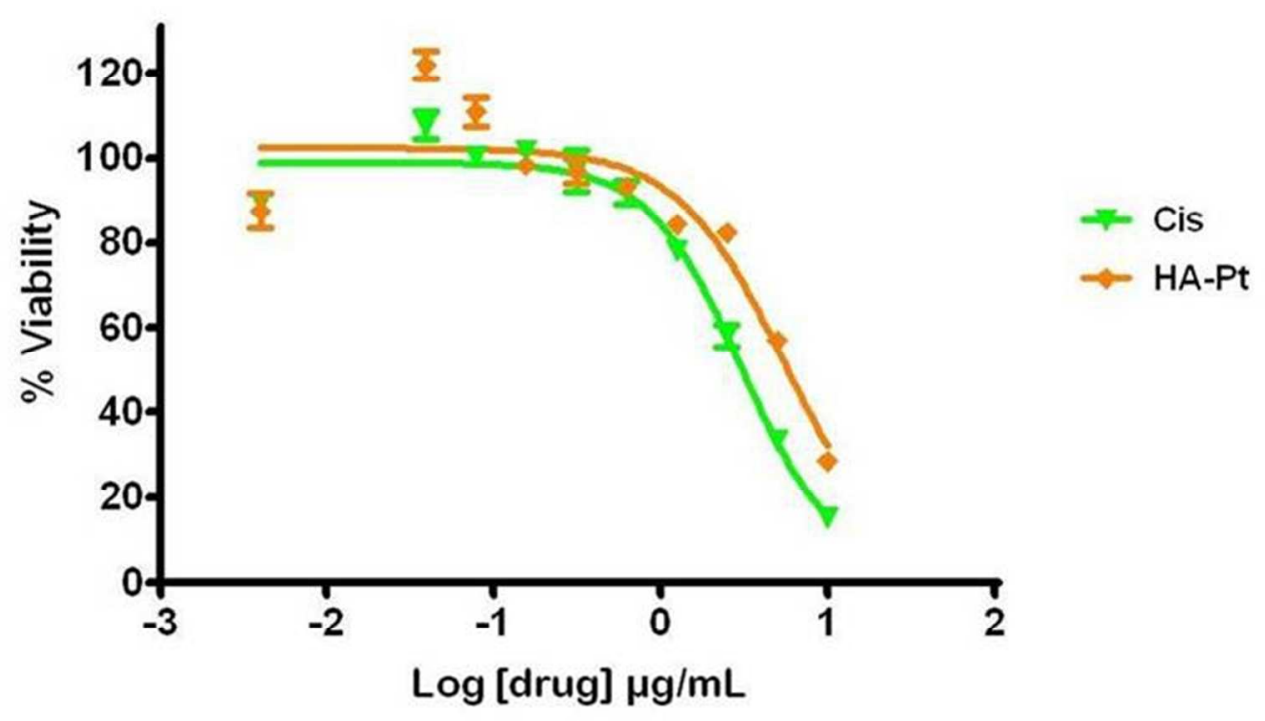

Figure 2. Best-fit non-linear dose response curves showing dose-dependent decrease in cell viability of UMSCC -12 cells with drug treatment.

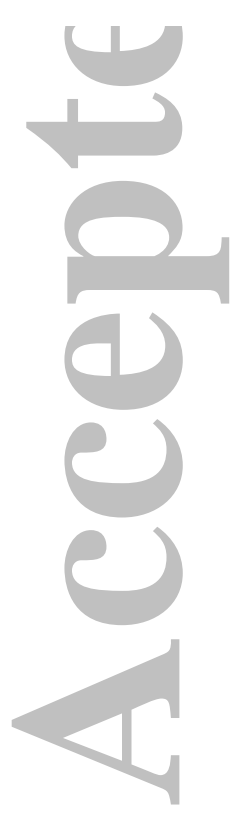




\section{Tumor Volume Curves}

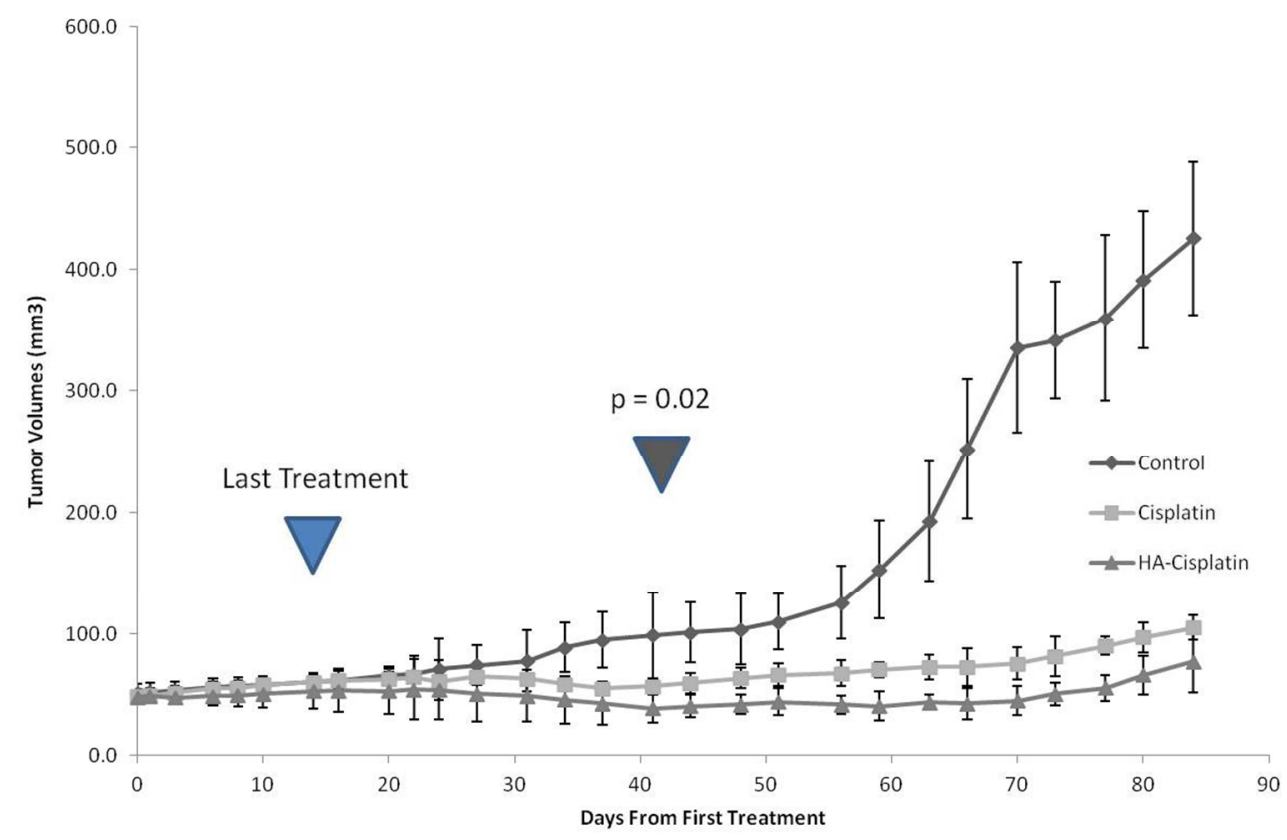

Figure 3. Tumor response curves for the three treatment arms.

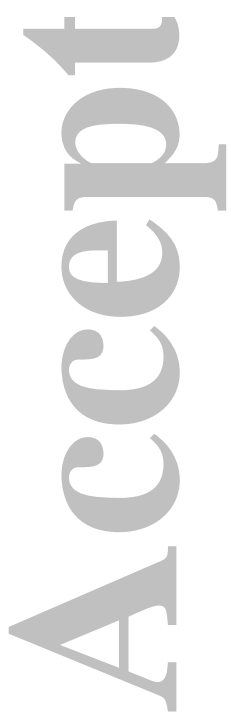
$205 \times 149 \mathrm{~mm}(150 \times 150$ DPI $)$ 


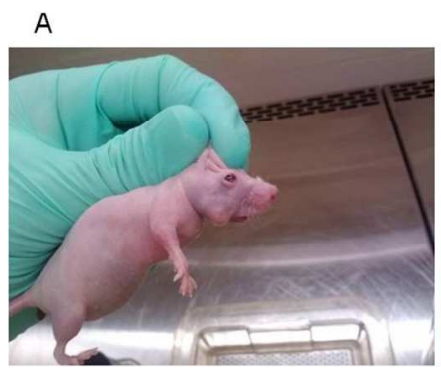

B
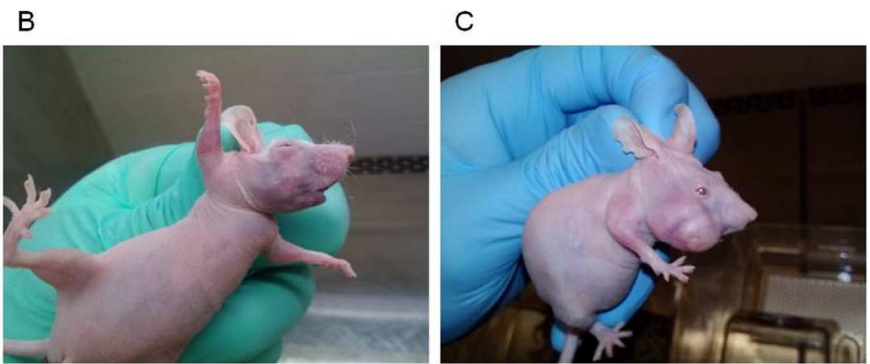

Figure 4. Examples of subject mice. A, a cisplatin mouse early after completion of treatment. B, a HAcisplatin mouse with partial response. C, a control mouse showing uninhibited tumor growth.

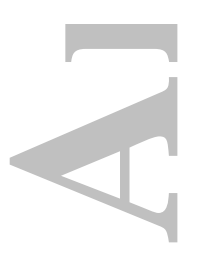
$233 \times 66 \mathrm{~mm}(150 \times 150 \mathrm{DPI})$

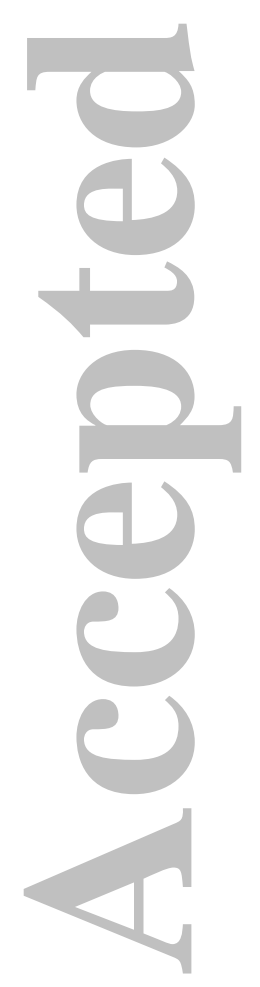




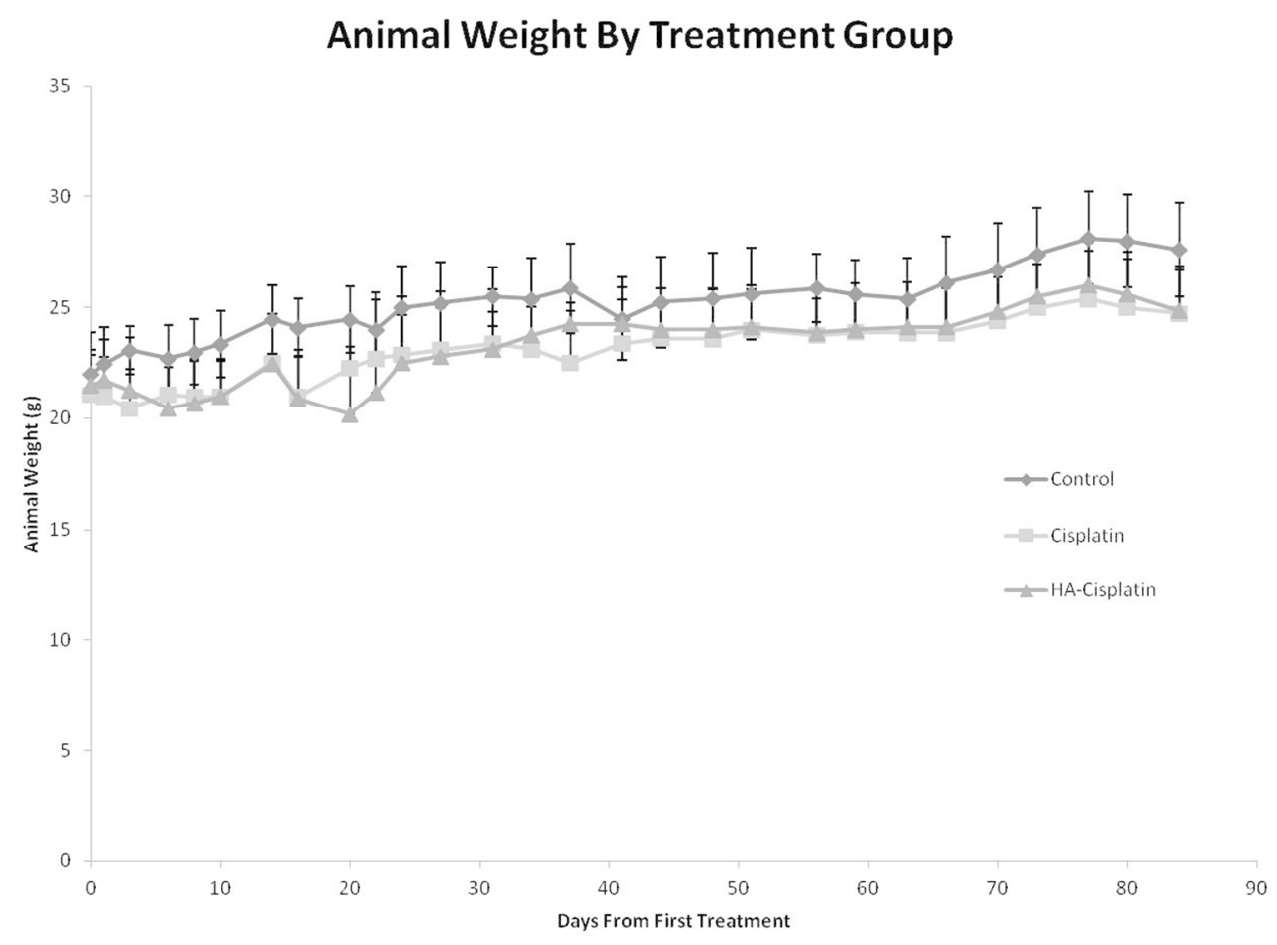

Figure 5. Weights of animal subjects monitored throughout the study. $216 \times 161 \mathrm{~mm}(150 \times 150 \mathrm{DPI})$ 


\section{Western blot: Early Post-Treatment}

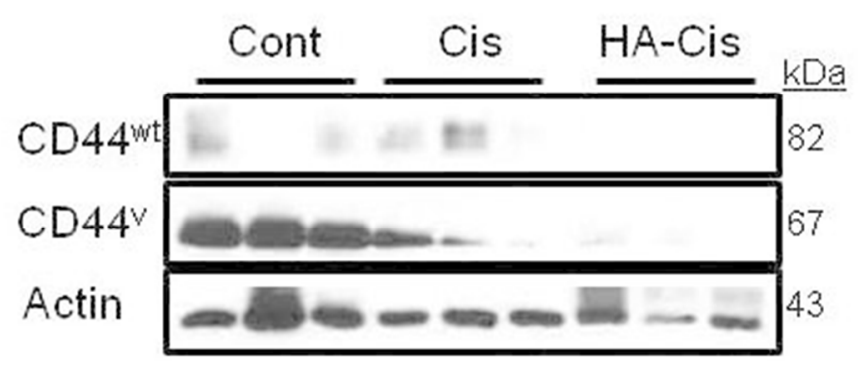

Figure 6. Western blot from early post-treatment group demonstrating reduction of CD44 expression in the two treatment arms, but to greater degree in HA-cisplatin.

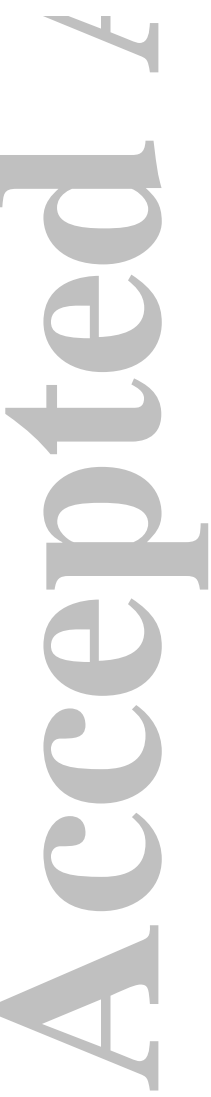


A

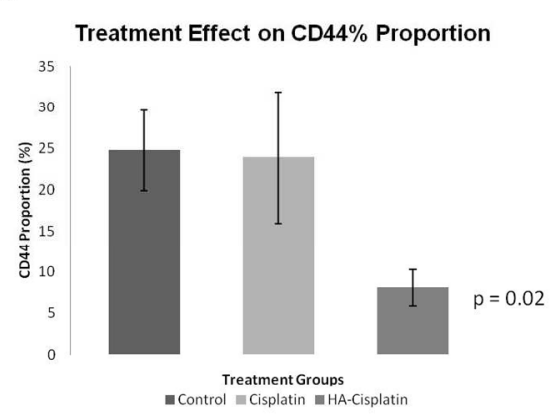

B
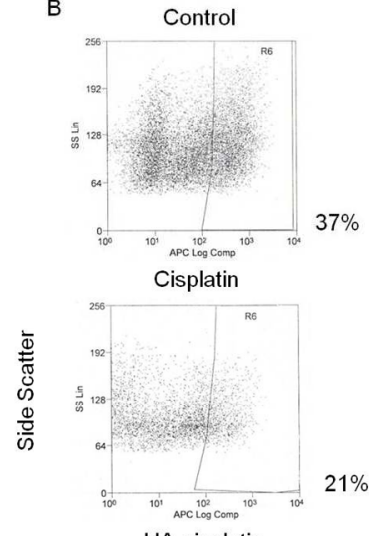

HA-cisplatin

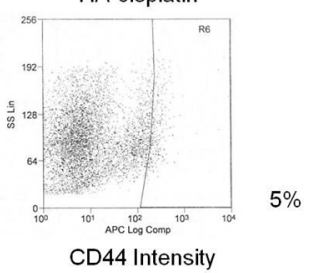

Figure 7. A, Flow cytometry data from end-of-study group demonstrating significant reduction of CD44 expression in the HA-cisplatin. B, Examples of flow cytometry diagrams from each group.

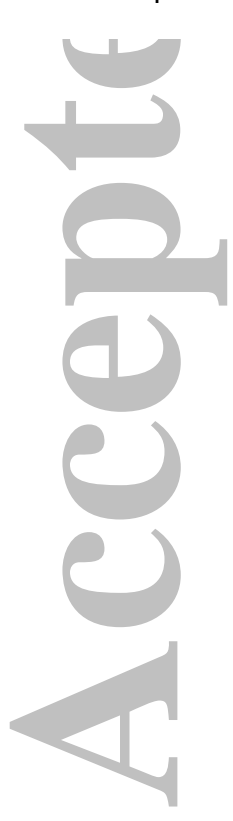
$284 \times 182 \mathrm{~mm}(150 \times 150 \mathrm{DPI})$ 


\section{Western blot: End-of-Study}

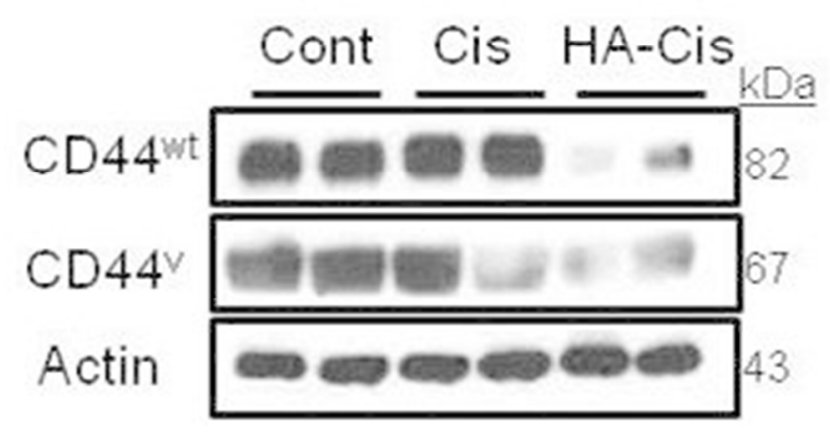

Figure 8. Western blot from end-of-study group demonstrating reduction of CD44 expression in HA-cisplatin group. $89 \times 47 \mathrm{~mm}(131 \times 131 \mathrm{DPI})$

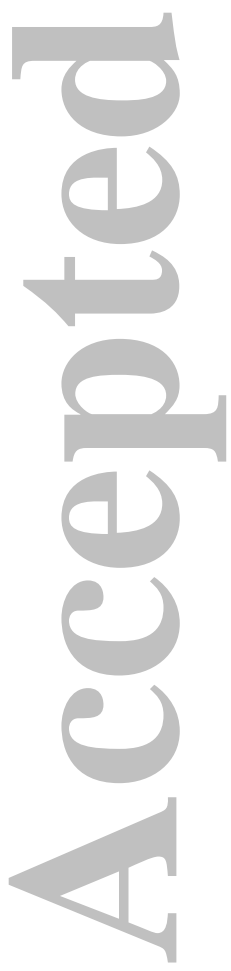

